\title{
Supporting Information Manuscript es061018y
}

Characterizing the redox status in three different forested wetlands with geochemical data

Christine Alewell ${ }^{1,2^{*}}$, Sonja Paul ${ }^{1,3}$, Gunnar Lischeid ${ }^{1}$, Kirsten Küsel ${ }^{1,4}$, Matthias Gehre $^{5}$

${ }^{1}$ BITÖK, University of Bayreuth, Dr.-Hans-Frisch-Str. 1-3, D-95440 Bayreuth, Germany

${ }^{2}$ present and *corresponding address:

Environmental Geosciences

University of Basel

Bernoullistr. 30

$\mathrm{CH}-4056$ Basel, Schwitzerland

phone: 0041-61-2670477

Fax: 0041-61-2670479

Email: christine.alewell@unibas.ch

${ }^{3}$ present address:

Institute of Soil Science and Forest Nutrition, Georg-August University Göttingen, Büsgenweg 2, D-37077 Göttingen, Germany

${ }^{4}$ present address:

Limnology Research Group, Institute of Ecology, Friedrich Schiller University

Jena, Carl-Zeiss-Promenade 10, 07745 Jena, Germany

${ }^{5}$ UFZ-Umweltforschungszentrum Leipzig-Halle, Permoserstr. 15, 04318 Leipzig, Germany

Number of pages: 5

Number of Tables: 1

Number of Figures: 3 
Table S1: Soil chemical characteristics. $\mathrm{Fe}_{\mathrm{d}}=$ dithionite extractable iron, $\mathrm{Fe}_{\mathrm{o}}=$ oxalate extractable iron. Data of the upland and the lowland fen are mean values (standard deviation) of $n=5$. Data of the seep are averages of duplicate soil samples.

\begin{tabular}{|c|c|c|c|c|c|c|c|c|c|c|c|}
\hline \multirow{2}{*}{$\begin{array}{l}\text { Field site } \\
\text { upland }\end{array}$} & $\begin{array}{c}\text { Depth } \\
(\mathrm{cm})\end{array}$ & \multicolumn{2}{|c|}{$\begin{array}{c}\mathrm{C}_{\text {org }} \\
\left(\mathrm{g} \mathrm{kg}^{-1}\right)\end{array}$} & \multicolumn{2}{|c|}{$\begin{array}{c}N_{\text {tot }} \\
\left(\mathrm{g} \mathrm{kg}^{-1}\right)\end{array}$} & \multicolumn{2}{|c|}{$\begin{array}{c}S_{\text {tot }} \\
\left(\mathrm{g} \mathrm{kg}^{-1}\right)\end{array}$} & \multicolumn{2}{|c|}{$\begin{array}{c}\mathrm{Fe}_{\mathrm{d}} \\
\left(\mathrm{g} \mathrm{kg}^{-1}\right)\end{array}$} & \multicolumn{2}{|c|}{$\begin{array}{c}\mathrm{Fe}_{\mathrm{o}} \\
\left(\mathrm{g} \mathrm{kg}^{-1}\right)\end{array}$} \\
\hline & $5-10$ & 346.1 & $(82.5)$ & 13.8 & (2.7) & 6.0 & (3.2) & 3.3 & (3.7) & 2.2 & (2.3) \\
\hline & $10-20$ & 231.6 & (206.4) & 10.6 & (9.3) & 4.2 & (3.5) & 0.6 & $(0.4)$ & 0.5 & (0.3) \\
\hline & $75-90$ & 147.7 & (143.3) & 5.4 & (4.8) & 2.1 & (1.6) & 3.1 & (3.4) & 1.9 & (1.9) \\
\hline \multirow[t]{3}{*}{ seep } & $0-10$ & 372 & & 19.8 & & $3.4^{*}$ & & 2.1 & & 2.0 & \\
\hline & $10-20$ & 429 & & 24.4 & & $2.9^{*}$ & & 3.6 & & 3.0 & \\
\hline & $20-30$ & 347 & & 19.2 & & $3.5^{\star}$ & & 2.6 & & 2.3 & \\
\hline \multirow[t]{3}{*}{ lowland } & $5-10$ & 301.5 & (102.6) & 13.4 & (2.9) & 3.2 & (1.2) & 13.5 & (11.7) & 10.8 & (9.7) \\
\hline & $10-20$ & 266.2 & $(96.5)$ & 210.4 & (2.9) & 2.8 & (1.5) & 6.9 & $(7.2)$ & 5.5 & (5.4) \\
\hline & $75-90$ & 10.9 & $(8.5)$ & 0.6 & (0.3) & $<$ & nd & 0.8 & $(0.6)$ & 0.6 & (0.5) \\
\hline
\end{tabular}

* Data from Kaiser, M. Bestimmung von Sulfat-Reduktionsraten mit der ${ }^{35} \mathrm{SO}_{4}{ }^{2-}$-Methode in säurebelasteten Quelleinzugsgebieten des Frankenwaldes und des Fichtelgebirges (Nordostbayern). Diplomarbeit an der Universität Bayreuth 1996. 


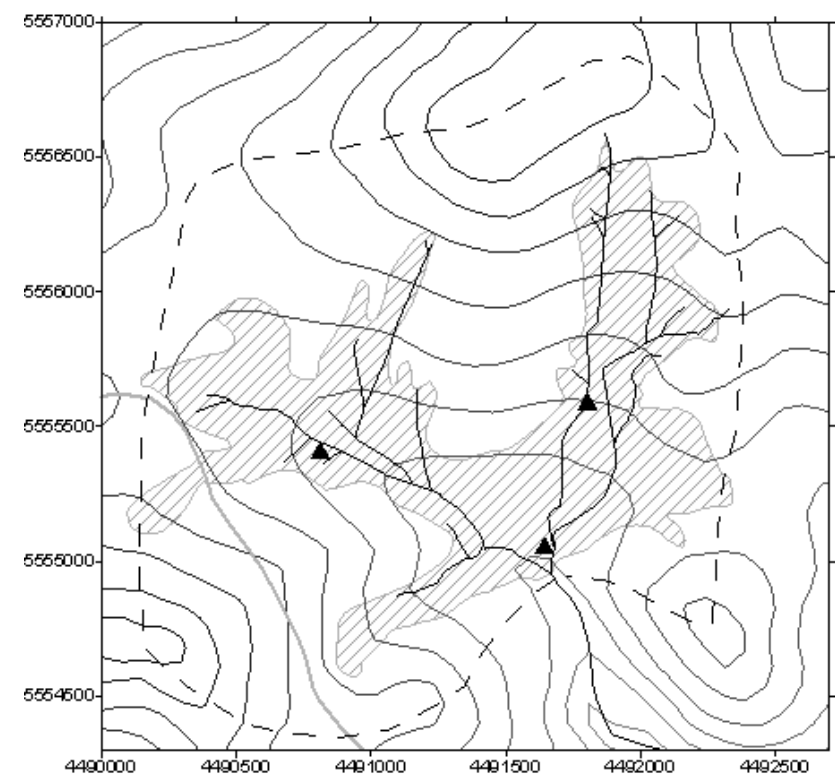

Figure S1: Map of the catchment Lehstenbach (left) and map of the installed lysimeters and piezometers at the fens (below). Shaded areas in the catchment map represent wetland areas.
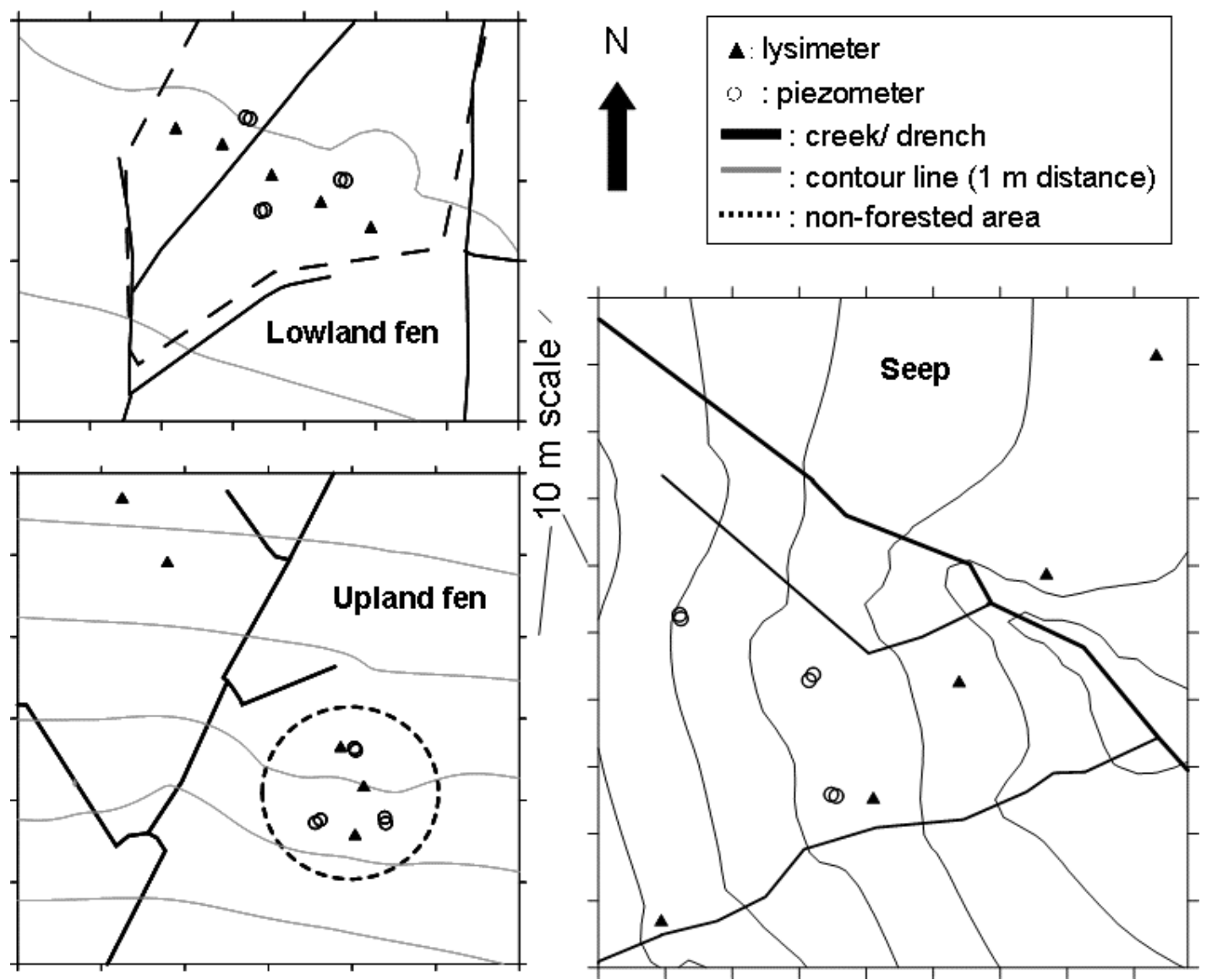

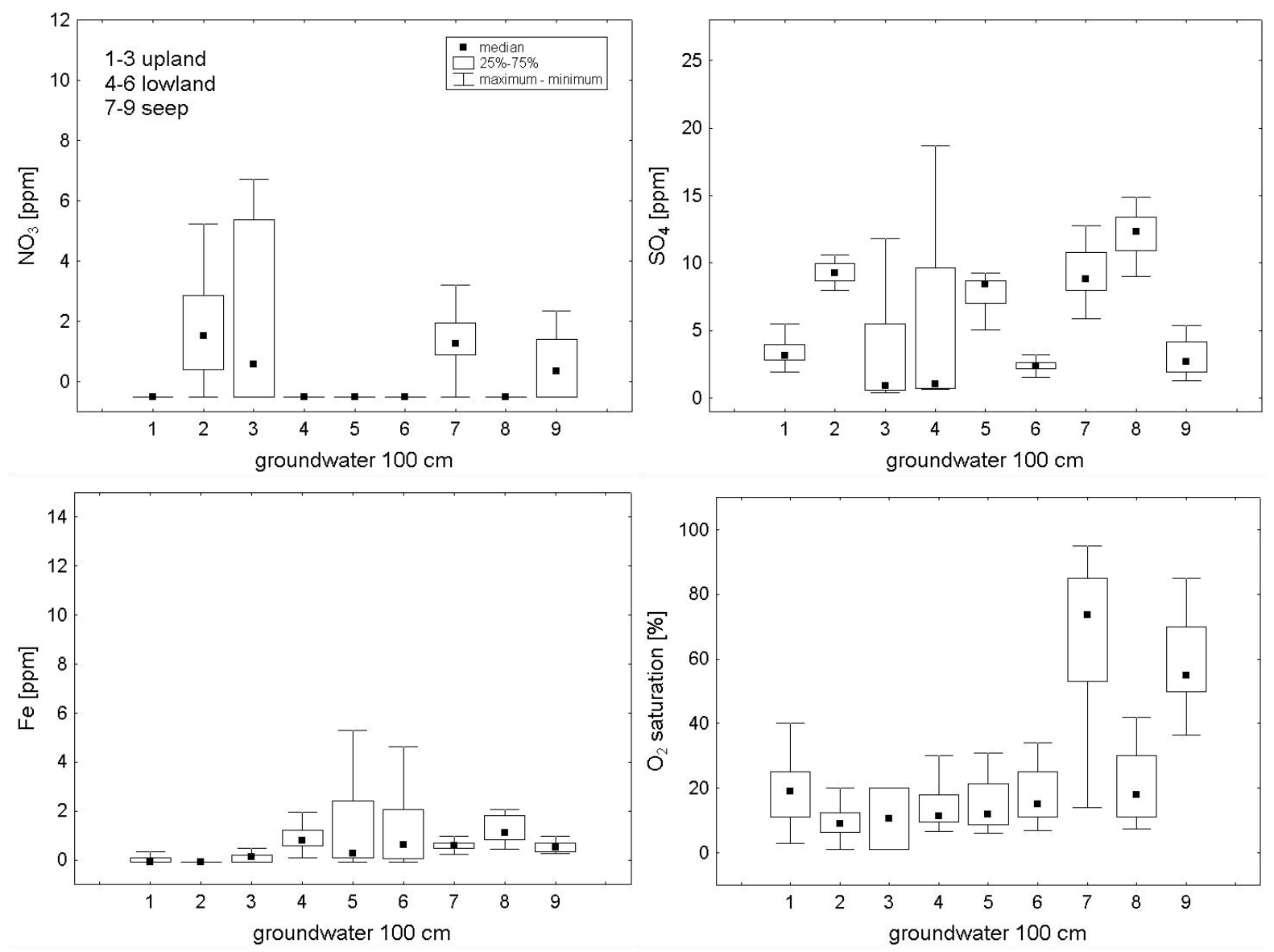

Figure S2: Piezometer concentrations over the measurement period (Jan 01 Dec02) at $100 \mathrm{~cm}$ depth at the fens. 

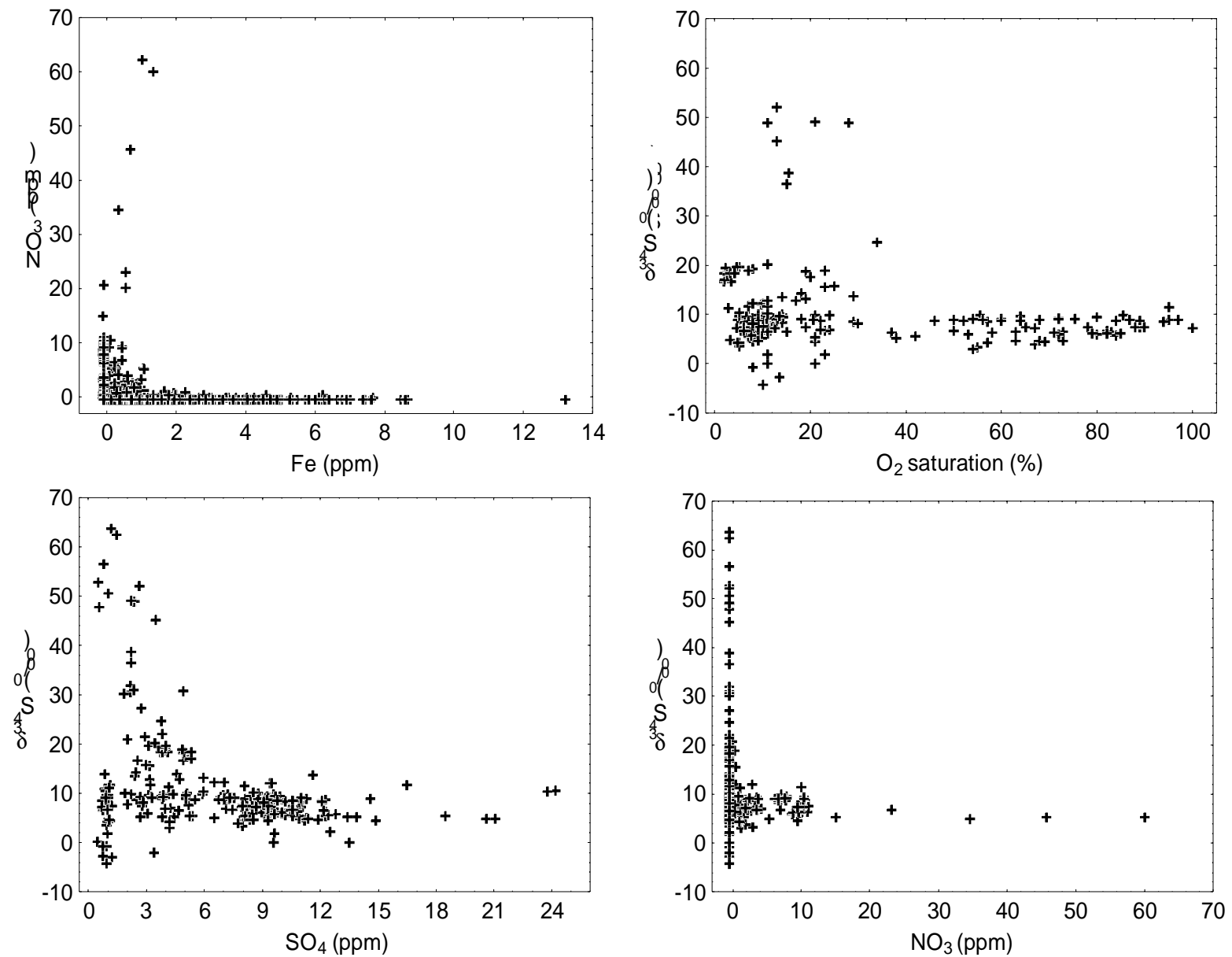

Fig. S3: Relationship of redox parameters. Each subplot presents all measured data from soil solutions and groundwater at the three fens. 